\title{
O trabalho e a formação do agente comunitário de saúde
}

\section{The work and formation of the communitary health agent}

\author{
${ }^{1}$ Lucrecia Helena Loureiro lucreciahelena@gmail.com \\ ${ }^{1}$ Mayara Athanazio Diogo \\ ${ }^{1}$ Thabata Braga \\ ${ }^{1}$ Flavio Vaz Machado \\ 2 Paulo Sérgio Marcellini \\ 2 Teresa Tonini
}

\section{RESUMO}

A Estratégia Saúde da Família (ESF) é um modelo assistencial que prioriza as ações de saúde em um contexto multiprofissional na atenção básica onde uma equipe formada por profissionais de diferentes áreas ajuda a enfrentar com qualidade os muitos determinantes sociais de saúde. Atualmente, não existe mais o foco em somente uma área do conhecimento capaz de dar respostas à complexidade humana, faz necessária a intervenção multiprofissional, haja vista as tantas desigualdades sociais. Acreditamos que os saberes se estabelecem com as trocas. O estudo trata de um curso de capacitação de agentes comunitários de saúde (ACS). Teve como metas auxiliar na capacitação destes profissionais que passaram pelo processo seletivo, efetivado no município de Angra dos Reis, em junho de 2013 e como estratégia eliminatória do concurso a não participação em $100 \%$ nas atividades teóricas e praticas do treinamento. Como objetivo geral preparar Agentes comunitários de Saúde para o desenvolvimento de ações de promoção de saúde, prevenção e atenção, enquanto componentes da equipe de atenção primária. E como objetivos específicos: Discutir os fundamentos do SUS( Sistema Único de Saúde), Refletir sobre a Saúde como Direito do Cidadão, conhecer os princípios e diretrizes legais relativas à Saúde; Esclarecer os conceitos de território, área, micro área, família e domicílio; Conhecer o conceito de trabalho em equipe em saúde; Identificar o papel do ACS como elo entre a comunidade e a Unidade de saúde; Identificar a atribuição do agente comunitário de saúde. Estudo retrospectivo, descritivo e observacional de um treinamento de Agentes Comunitários de Saúde no Município de Angra dos Reis. Os participantes foram reunidos em dois grupos, turma em horário parcial e integral, para comparação: faixa etária, gênero, escolaridade, avaliação do curso e dos facilitadores. Os testes de Qui- quadrado e Exato de Fisher, a ANOVA e o teste de Kruskal-Wallis foram utilizados na análise dos grupos. Os achados revelaram que a capacitação atingiu os objetivos propostos e concluiu-se que a capacitação deve ser utilizada para subsidiar o processo de ensino-aprendizagem dos Agentes Comunitários de Saúde, bem como para adequar, unificar e padronizar o atendimento desses agentes da Atenção Básica de Saúde no Município de Angra dos Reis.

Palavras-chave: Estratégia Saúde da Família, Agente Comunitário de Saúde, Ensino

\section{ABSTRACT}

The Family Health Strategy (ESF) is a care model that prioritizes health actions in a multidisciplinary context in primary care where a team of professionals from different areas helps to face with quality the many social determinants of health. Currently, there is more focus on only one area of knowledge able to respond to human complexity and the multi-professional intervention is necessary, given the many social

1 Centro Universitário de Volta Redonda-UniFOA

2 Universidade Federal do Estado do Rio de Janeiro- Unirio 
inequalities. We believe that knowledge is established with exchanges. The study is a training course for community health agents (ACS). The goals were assisting in the training of these professionals who who attended the selection process, which was effected in the municipality of Angra dos Reis, in June, 2013, and it had as a round strategy of the competition the non-participation at 100\% in the theoretical and practical training activities. The overall goal was to prepare Community Health Workers to develop actions of health promotion, prevention and care, as components of the primary care team. It also had the following specific objectives: Discussing the fundamentals of SUS (Single Health System), reflecting on Citizen's Right to Health, meeting the legal principles and guidelines relating to health; clarifying the concepts of territory, area, micro area, family and home; knowing the concept of teamwork in health; Identifying the role of the ACS as a liaison between the community and the health unit; Identifying the allocation of community health agent. Retrospective, descriptive and observational study of a training of Community Health Workers in the municipality of Angra dos Reis. The participants were divided into two groups, class into full and part time, for comparison: age, gender, education, and evaluation of the course and facilitators. The Chi-square test and Fisher's exact, the ANOVA and Kruskal-Wallis test were used in the group analysis. The findings revealed that the training achieved its objectives and it was concluded that training should be used to support the teaching and learning of Community Health, as well as to adapt, to unify and standardize the care of these agents for primary care in the health in the municipality of Angra dos Reis.

Keywords: Family Health Strategy, Community Health Agents, Teaching.

\section{INTRODUÇÃO}

A inserção do agente comunitário de saúde ( ACS) na rede do Sistema único de Saúde ( SUS) tem suscitado polêmica a respeito da forma mais adequada de relação de trabalho que deve ser adotada para que se possa contar de modo sustentável com esse tipo de recurso humano ( NOGUEIRA,2000).

Reconhecidamente o trabalho do agente comunitário de saúde lida com demandas relativas às condições de vida, à política, à cidadania e à organização dos grupos e suas relações, incluindo a família. Desta forma, urge o conhecimento do campo da saúde, além dos saberes que perpassam o cotidiano da sua atuação prática e esse saber precisa estar sistematizados na formação dos ACS. Para a realização de um trabalho de qualidade pelos ACS no campo da saúde, independentemente da realidade local que esses profissionais atuam.

Na década de 80, iniciou-se um clima de reestruturação do modelo de saúde vigente, e esta reforma do modelo de saúde ocorreu com a criação do Sistema único de Saúde (SUS). Com o novo modelo, onde as ações de saúde na lógica organizativa dos serviços, com universalidade, integralidade, como diretrizes básicas da porta de entrada do sistema, priorizam as ações preventivas em detrimento as curativas.

Em 1994, o Ministério da Saúde, decidiu reorganizar o modelo de atenção a saúde vigente nos pais e instituiu o Programa de Saúde da Família. A grande maioria dos municípios aderiu ao programa e iniciaram com a nova política de atendimento a população. Para a implantação do programa, foi necessária a contratação de uma equipe multidisciplinar, composta de médico, enfermeiro, técnicos de enfermagem e Agentes Comunitários de Saúde, em busca de uma efetivação e sofisticação da Atenção Primária a Saúde.

Com um novo olhar para a saúde da população o foco passou a ser a Saúde e não a doença, a família e não o individuo. A comunidade passou a ser assistida por uma equipe e não somente por um profissional, desta forma o PSF, propõe à melhoria da qualidade de vida dessas pessoas.

Atualmente, em sua grande maioria, os ACS estão submetidos a relações informais de trabalho. No Município de Angra dos Reis esses trabalhadores eram recrutados de forma inadequada, para uma função que exige 
um relacionamento estreito e permanente com a comunidade, desta forma, como alternativa de adequar sua estrutura organizacional, foi proposta pelo Secretário Municipal de Saúde a admissão desses profissionais ao quadro de servidores públicos através de um concurso em regime CLT, ocorrida em três etapas: $1^{\text {a }}$ etapa prova teórica, $2^{\mathrm{a}}$ etapa curso Introdutório e a $3^{\mathrm{a}}$ etapa uma atividade prática orientada no curso Introdutório. O curso Introdutório fundamentava-se especificamente para preparar e adequar esses profissionais para atuarem na Rede de Saúde da cidade e a não participação em 100\% nas atividades teóricas e praticas ao treinamento era eliminatório.

O objetivo do estudo foi relatar a experiência do curso, caracterizar os agentes comunitários de saúde, avaliar e analisar quanti-qualitativamente o curso sob a ótica dos participantes.

\section{ABORDAGEM METODOLÓGICA}

Trata-se de um estudo retrospectivo, descritivo e observacional de Agentes Comunitários de Saúde no período de junho a agosto de 2013 na Fundação de Saúde de Angra dos Reis ( FUSAR).

Foi realizada análise bivariada para comparar as características dos grupos participantes. O teste de Quiquadrado $\left(x^{2}\right)$ e o teste Exato de Fisher foram empregados na comparação de proporções. Para as variáveis quantitativas utilizaram-se a análise de variância ( ANOVA), e o Teste de Kruskal-Wallis.

A Fundação de Saúde de Angra dos Reis - FUSAR, pessoa jurídica de direito público, com sede na Rua Almirante Machado Portela nº 85, Balneário, Angra dos Reis/RJ, representado por seu Presidente , no uso das atribuições que lhe foram conferidas pela Lei $n^{\circ}$ 1509, 30 de dezembro de 2004, tornou pública através do processo administrativo $\mathrm{N}^{\circ}$ 1437/2013, realização de processo seletivo, visando a contratação de empregados públicos agentes comunitários de saúde, para suprir carência nos termos da Lei Municipal No 1.941, de 30 de abril de 2008.

O Processo Seletivo destinou-se à seleção de agentes comunitários de saúde, para efetuar cobertura de vagas existentes no quadro de lotação de empregados públicos da Secretaria Municipal de Saúde, a serem regidos pela Consolidação das Leis do Trabalho. Como os requisitos básicos para a contratação nos cargos são, cumulativamente, os seguintes: residir na área da comunidade em que atuar desde a data da publicação do edital do processo seletivo público; haver concluído o ensino fundamental; ter sido aprovado e classificado no Processo Seletivo; haver concluído, com aproveitamento, curso de qualificação básica de formação, que será ministrado pela Secretaria Municipal de Saúde quando da convocação dos candidatos.

O processo seletivo para contratação destes profissionais ocorreu em 02 etapas, sendo a $1^{\text {a }}$ etapa em junho de 2013, com prova objetiva com 30 questões distribuídas em 10 questões de português, 10 de matemática e 10 especificas da área de atuação; a $2^{\mathrm{a}}$ etapa ocorreu em agosto do mesmo ano, através de um curso introdutório organizado pela Atenção Básica, com carga horária de 40h, distribuída em 20h teóricas e 20h práticas, sendo pré-requisito para investidura no cargo de ACS conforme consta no edital.

Foram 40h de treinamento em 10 encontros no horário de $8 \mathrm{~h}$ às 17h, as aulas ocorreram no auditório do Hospital Municipal. O curso foi organizado pela Superintendência da Atenção Básica do Município de Angra dos Reis, ministrado pelas autoras do artigo.

No primeiro dia de curso os candidatos foram subdivididos em 03 turmas, 01 turma em horário integral (28 candidatos), 01 turma no período da manhã (21candidatos) e 01 turma no horário da tarde (22 candidatos), num total de 71 candidatos participantes. 
Todos os participantes receberam uma apostila no primeiro dia de aula, com todo o conteúdo programático. Utilizaram-se como estratégia de ensino, vídeos motivacionais, slides, dinâmicas, o grupo foi estimulado pelas monitoras a utilizar criatividade, com a solicitação de representação gráfica a utilizar desenhos família e a confeccionar um mapa territorial, essa estratégia busca a comprovação da residência do candidato.

Ressaltamos que um pré-requisito para investidura ao cargo de ACS é residir há pelo menos seis meses no bairro que pleiteia vaga, porém, esta atividade gráfica quando solicitada aos candidatos, permite aos monitores a observação do comportamento do candidato e suas fragilidades diante da atividade proposta. Desenhar um mapa requer conhecimento detalhado do bairro.

Concluído as aulas teóricas, os candidatos foram redistribuídos entre as ESF do município, os mesmos deveriam cumprir $12 \mathrm{~h}$ de atividade prática. Foram solicitadas duas tarefas como requisitos avaliativos: Como primeira atividade a realização de uma Visita Domiciliar (VD) com preenchimento das fichas (A e B) e a segunda uma Sala de Espera com temas pré-definidos (amamentação e tabagismo); Foi solicitado registro fotográfico da unidade para comprovação do comparecimento e entrega de todo material preenchido no último dia do encontro com as monitoras.

Ao final do treinamento, decidimos publicar um artigo, relatando essa experiência. Para realização do estudo solicitamos, inicialmente, a permissão da Secretaria Municipal de Saúde de Angra dos Reis, após concordância as facilitadoras solicitaram aos candidatos que participaram do curso, autorização escrita onde todos foram esclarecidos sobre os objetivos da pesquisa, concordaram em participar da mesma assinando o Termo de Consentimento Livre e Esclarecido, para ser submetido à aprovação do Comitê de Ética em Pesquisa do Centro Universitário de Volta Redonda, Decreto n ${ }^{\circ} 93.933$ de 13/01/1987, Resolução 196/96 do Conselho Nacional de Saúde (BRASIL, 1996). O projeto foi aprovado pelo Comitê de Ética em Pesquisa, processo número 120/2011, e não houve fontes externas de financiamento.

\section{RESULTADOS E DISCUSSÃO}

\section{1 Perfil sócio-demográfico e educacional dos ACS}

A analise estatística descritiva dos dados foi processada pelo software de domínio público criado pelo CDC(Centers for Disease Control and Prevention, em português Centro para o controle e prevenção de doenças) voltado a área da saúde na parte de epidemiologia, denominado Epi info. Foi realizada análise bivariada para comparar as características dos grupos participantes. O teste de Qui- quadrado( $\left.\mathrm{x}^{2}\right)$ e o teste Exato de Fisher foram empregados na comparação de proporções. Para as variáveis quantitativas utilizaram-se a análise de variância ( ANOVA), e o Teste de Kruskal-Wallis.

A Tabela 1. Na analise estatística dos dados apresenta o perfil sócio-demográfico dos ACS, que se concentra na faixa etária de 18 a 28 anos com 31\% , 29 a 39 anos 8,5\% , 40 a 50 anos 2,6\% e 51 a 60 anos somente $1,3 \%$. No presente estudo, verificou-se quanto à distribuição por sexo, observou-se que $28,3 \%$ dos candidatos ao cargo de ACS eram do sexo feminino, com ensino médio. Relativiza, neste caso, a ideia da ESF no município de Angra, estar se constituindo para as mulheres como inserção inicial no mercado de trabalho.

Observa-se nesta tabela que dos 71 candidatos que participaram do treinamento, sua grande maioria, 93\% concordaram em participar da pesquisa. 
Tabela1. Distribuição dos ACS, por idade, sexo, escolaridade e formação profissional. Angra dos Reis-RJ,ago.2013.

\begin{tabular}{ccc}
\hline Variáveis & $\begin{array}{c}\text { Horário Integral } \\
\mathrm{n}(\%)\end{array}$ & $\begin{array}{c}\text { Horário Meio Período } \\
\mathrm{n}(\%)\end{array}$ \\
\hline SEXO & $18(66,6)$ & $25(64,1)$ \\
Feminino & $09(33,4)$ & $14(35,9)$ \\
Masculino & & \\
IDADE (anos) & $17(63,0)$ & $30(76,9)$ \\
$18-28$ & $08(29,6)$ & $05(12,8)$ \\
$29-39$ & $02(7,4)$ & $02(5,1)$ \\
$40-50$ & $00(0,0)$ & $02(5,1)$ \\
$50-60$ & & $07(17,9)$ \\
ESCOLARIDADE & $01(3,7)$ & $28(71,7)$ \\
Fundamental & $21(77,7)$ & $04(10,2)$ \\
Médio & $05(18,5)$ & 39 \\
Superior & 27 & \\
\hline Total & & \\
\hline
\end{tabular}

Fonte: Boletim de Avaliação de Treinamento

A análise da avaliação demonstrou que os dois grupos avaliaram como ótimo ( 75,7\%) e bom( 24,3\%) o curso ministrado pelas autoras do artigo. Quanto ao desempenho das facilitadoras como ótimo ( 91,0\%) e bom( 9,0\%), conforme demonstra tabela.2.

Tabela2. Avaliação do curso e dos Facilitadores por nível de escolaridade. Angra dos Reis-RJ,ago.2013.

\begin{tabular}{|c|c|c|c|}
\hline Variáveis & $\begin{array}{c}\text { Horário Integral } \\
\text { n ( \%) }\end{array}$ & $\begin{array}{c}\text { Horário Meio } \\
\text { Período } \\
\text { n (\%) }\end{array}$ & P-valor \\
\hline \multicolumn{4}{|c|}{ AVALIAÇÃO DO CURSO* } \\
\hline Ótimo & $21(78,0)$ & $29(74,3)$ & \\
\hline Bom & $06(22,0)$ & $10(25,7)$ & 0,7494 \\
\hline \multicolumn{4}{|c|}{ AVALIAÇÃO DOS } \\
\hline \multicolumn{4}{|c|}{ FACILITADORES** } \\
\hline Ótimo & $24(89,0)$ & $36(92,4)$ & 0,6822 \\
\hline Bom & $03(11,0)$ & $03(7,6)$ & \\
\hline \multicolumn{4}{|c|}{ ESCOLARIDADE } \\
\hline Fundamental & $01(3,8)$ & $07(18,0)$ & 0,083 \\
\hline Médio & $21(77,7)$ & $28(72,0)$ & 0,001 \\
\hline Superior & $05(18,5)$ & $04(10,0)$ & 0,003 \\
\hline Total & $27(100,0)$ & $39(100,0)$ & \\
\hline
\end{tabular}


Foram aplicados os testes de qui-quadrado para verificar se existia diferença significativa $(\mathrm{p}<0,05)$ entre a avaliação do curso nos períodos integral e meio período, e teste Exato de Fisher (já que duas das células tem n inferior a 5) para verificar se existe diferença entre a avaliação dos facilitadores. Em ambos os casos não houve diferença significativa ( $p>0,05)$ o que permite concluir que estatisticamente as avaliações foram similares entre os períodos. Para escolaridade não foi possível fazer teste estatístico já que as células apresentavam ns inferiores a 5 e o testes exato de Fisher só se aplica em tabelas 2 por 2.

Gráfico 1. e 2, apresentam o desempenho dos facilitadores, autores da pesquisa.

\section{Gráfico 1: Avaliação dos Facilitadores realizado pela turma integral.} Angra dos Reis,dezembro de 2013

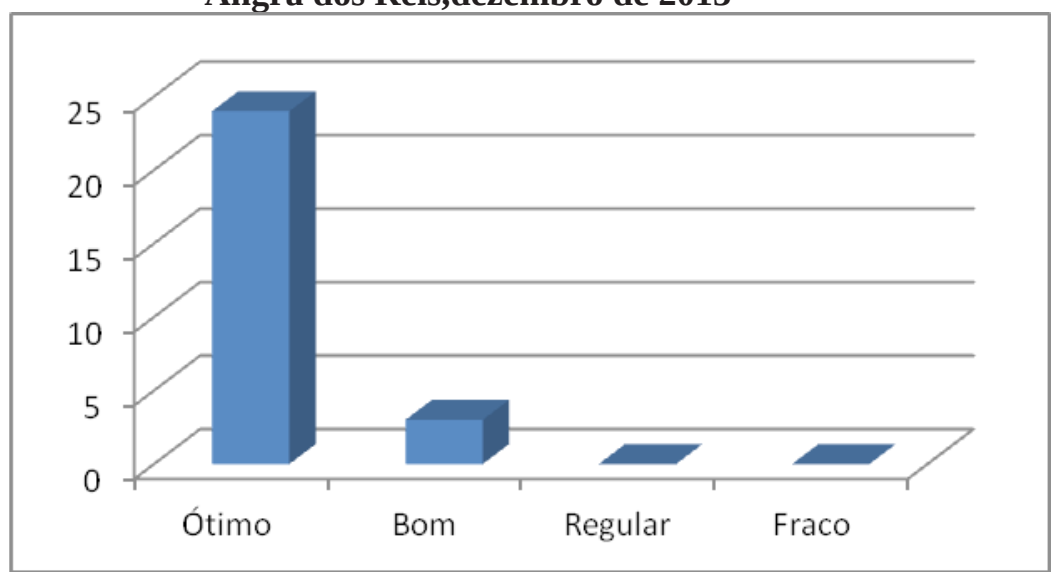

Fonte: Boletim de Avaliação de Treinamento- turma integral

\section{Gráfico 2: Avaliação dos Facilitadores realizado pela turma de um período.} Angra dos Reis,dezembro de 2013

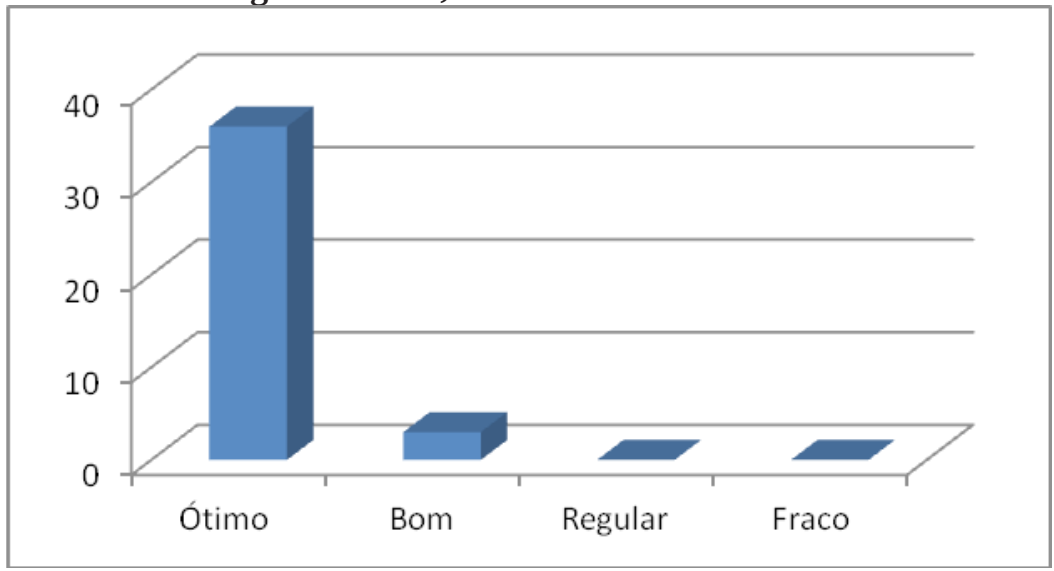

Fonte: Boletim de Avaliação de Treinamento- turma meio período

Os ACS mostraram-se animados para responder sobre o curso e tinham a consciência de que a avaliação serviria para melhorar o serviço de saúde que utilizam e irão trabalhar, muitos descreveram e contribuíram, onde extraímos algumas considerações interessantes, tais como:

“O curso foi bastante dinâmico, possuindo muitas informações acerca do trabalho prático do agente comunitário de Saúde”.( IF26) 
"O curso me possibilitou enxergar o trabalho do ACS de uma maneira diferente, foi um mergulho em outra realidade, onde a base é o respeito pelo outro.(IF 3)

"O curso foi muito bom, pois através dele passamos a conhecer o trabalho do ACS, sua importância e contribuição para a comunidade”.( IF 25)

"Essa oportunidade foi excelente, para os novatos, pois podemos aprender na prática as funções que iremos desempenhar na unidade”.( IF 22)

Percebemos nos registros o processo como positivo para melhoria da qualidade do serviço. Entendemos que faz necessária uma Educação permanente direcionada ao ACS com poucos recursos de custeio são algumas observações que fazemos.

\section{CONSIDERAÇÕES FINAIS}

O ACS pode ser visto como um trabalhador genérico, que realiza tarefas não restritivas ao campo da saúde. No entanto, a especial vulnerabilidade econômica dessa categoria de trabalhador justifica a adoção, na política de recursos humanos do SUS, de uma diretriz de observância dos benefícios sociais dados pela legislação de proteção ao trabalho.

Nessa perspectiva, há necessidade de desenvolvimento e incorporação de tecnologias que apoiem a identidade do ACS, quando se colocam em questão o trabalho e o saber do agente comunitário de saúde, integrando as diferentes dimensões de sua atuação, parece-nos muito atual.

No que se refere à qualificação do ACS, apesar do Ministério da Saúde definir as principais diretrizes para sua formação, na prática esse processo ainda é muito diversificado. Acreditamos que na qualificação reside a força e a riqueza da presença desse ator em uma equipe de saúde.

A qualificação dos trabalhadores permite uma nova visão do processo de trabalho, a necessidade de capacitação dos Agentes Comunitários de Saúde, que estão iniciando sua vida profissional em uma atividade, simples e ao mesmo tempo complexa. O ACS, querendo ou não participa e circula dentro de uma comunidade, das casas, convivem com o cotidiano da comunidade o qual faz parte.

O treinamento dos agentes vem ao encontro das políticas de saúde para melhorar a atenção de saúde local. Percebemos que as oficinas de sensibilização ocorrida com os ACS antigos para apresentação dos novos agentes, permitiram a compreensão do programa e fizeram as coisas começarem a mudar. Agora há integração entres as equipes existentes e os recém-chegados, os agentes trabalham juntos em prol da atenção básica, em articulação ao programa contando com o apoio institucional.

No Município, a lógica em relação à satisfação do profissional também sofreu alteração, a capacitação levou os profissionais a se preocuparem mais em organizar o trabalho de forma a dar aos usuários mais satisfação quando acessam os serviços oferecidos. Cada diretriz do treinamento foram estudados para que o maior número de informações possíveis fosse transmitido à equipe de ACS e apontamos que os dois períodos tiveram avaliações estatisticamente similares.

Antes, não existiam critérios para inclusão desses profissionais na ESF do Município, sua entrada era meramente indicação política. Afirmamos que o processo seletivo e o treinamento demonstraram credibilidade e maior responsabilidade a esses atores, sem contar com as melhorias reais na qualidade das ações e serviços de saúde, concluímos que faz necessário um programa de educação permanente desses profissionais. 
No caso dos Agentes Comunitários de Saúde, do Município de Angra dos Reis, era imprescindível a qualificação dos mesmos para assumirem o cargo. Acreditamos que toda empresa ou serviço que tenha como meta manter um padrão de qualidade, deve possuir um quadro de pessoal, com perfil de extrema competência e qualificados. Uma avaliação de desempenho, em particular dos ACS, acreditamos que nesses atores sociais reside a força e a riqueza de informações a respeito de uma comunidade.

Por fim, valer notar que as expectativas depositadas em torno da participação dos ACS, o cuidado da SAB que decidiu apostar em todo o conjunto e permitiu a formação desses atores para atuarem na atenção básica.

Entretanto, esperamos que o treinamento sirva para melhorar a qualidade dos serviços da Atenção Básica, temos a pretensão que a implantação do treinamento irá mudar a realidade das equipes e dos usuários do SUS dentro do município, já que o treinamento permitiu a compreensão do programa e esperamos que as coisas mudem, agora com apoio institucional, onde todos os envolvidos entenderam o desejo de melhoria da qualidade dos serviços prestados a comunidade. A construção e apropriação do conceito de trabalho em equipe, eles começaram a entender o que é esse trabalho e um dos pontos principais foi a união entre os participantes ao final do treinamento.

A avaliação do curso, descrita nos depoimentos dos ACS como resultado da relação destes com o ensinoaprendizagem do novo trabalho e como dispositivo regulador para os próximos cursos de capacitação, refletiu a existência e o uso das aptidões (aprendidas e improvisadas) do ACS e o cuidado comunitário. 


\section{REFERÊNCIAS}

BERNARDO, V. Desenvolvimento de programa multimídia aplicado ao ensino da medicina. Tese de Mestrado, EPM/UNIFESP, 1996.

BRASIL. Ministério da Saúde. Programa de Saúde da Família. Brasília, COSAC,1994.

BRASIL. Constituição Federal de 1988. Brasília: SENADO FEDERAL, 1988. . Lei 8080 de 19 de setembro de 1990. . Lei 8142 de 28 de setembro de 1990.

SILVA, J.A. O agente comunitário de saúde do Projeto QUALIS: agente institucional ou agente de comunidade? Dissertação (Mestrado em Saúde Pública ) Faculdade de Saúde Pública, Universidade de São Paulo. São Paulo, 2001.

LOTTA, G.S. Saber e Poder: Agentes Comunitários de Saúde Aproximando Saberes Locais e Políticas Públicas. 171f. Dissertação (Mestrado em Administração). Universidade de Administração de Empresas de São Paulo. 2006.

LOUREIRO, L.H. Ensino de gerência em saúde coletiva e a educação permanente dos profissionais da ESF: o uso do software educativo. 68f. Dissertação (Mestrado Profissional em Ensino em Ciências da Saúde e do Meio Ambiente) Volta Redonda - UniFOA, 2010.

MARTINES, W.R; CHAVES, E.C. Vulnerabilidade e sofrimento no trabalho do Agente Comunitário de Saúde no Programa de Saúde da Família. Rev Esc Enferm USP. 2007; 41(3): 426-33.

NOGUEIRA, R., RAMOS, Z.V.O. A vinculação institucional de um trabalhador sui generis - o agente comunitário de saúde. [Texto para discussão 735]. 2000. 33p. Disponível em <URL: http://www.ipea.gov.br >. Acesso em: jun 2012.

NUNES, M.O; TRAD, B.L; ALMEIDA, B.A; HOMEM, C.R; MELO M.C. AGENTE COMUNITÁRIO DE SAÚDE: personagem híbrido e polifônico. Cad. Saúde Pública, Rio de Janeiro, 18(6): 1639-1646 nov-dez, 2002.

SCHERER, M. D. A. et al. Rupturas e resoluções no modelo de atenção à saúde : reflexões sobre a estratégia saúde da família com base nas categorias Kuhnianas, Interface - Comunic., Saúde, Educ., v.9, n.16, p.53-66, set.2004/fev.2005.

SILVA, A.S; DALMASO. A.S.W. O AGENTE COMUNITÁRIO DE SAÚDE E SUAS ATRIBUIÇÕES: os desafios para os processos de formação de recursos humanos em saúde. Interface - Comunic, Saúde, Educ, v6, n10, p.75-94, fev 2002.

SOUZA, Rafaela A. e CARVALHO, Alysson M. Programa de Saúde da Família e Qualidade de Vida: um olhar da psicologia. Revista Estudos de Psicologia, 8(3), 515-523, 2003.

TEIXEIRA, C.F., PAIM, J.S. Planejamento e programação de ações intersetoriais para a promoção da saúde e da qualidade de vida. Rev. Adm. Pública, v.34, n.6, p.63-80, 2000. 